\title{
3D Printing Individualized Assisted Repair and Reconstruction of Irregular Bone Neoplastic Bone Defects: Exploration and Practice in Scapular Aneurysmal Cystic Bone Defects
}

Guochen Luo

Affiliated Zhongshan Hospital of Dalian University

yao Zhang ( $\square$ zhangyour@126.com )

Dalian University of Technology https://orcid.org/0000-0002-4725-111X

Xiahua Wang

Affiliated Zhongshan Hospital of Dalian University

Shuaishuai Chen

Affiliated Zhongshan Hospital of Dalian University

Dongyi Li

Affiliated Zhongshan Hospital of Dalian University

Mingyang Yu

Affiliated Zhongshan Hospital of Dalian University

Research article

Keywords: Irregular bone, Scapula, Aneurysmal bone cyst (ABC), 3D printing, Bone defect, Repair and Reconstruction

Posted Date: May 7th, 2021

DOI: https://doi.org/10.21203/rs.3.rs-477108/v1

License: (c) (i) This work is licensed under a Creative Commons Attribution 4.0 International License. Read Full License 


\section{Abstract}

Objective: To explore the clinical efficacy of using 3D printing individualized treatment plan in the auxiliary repair and reconstruction of irregular bone tumor bone defect.

Methods \Seven patients with aneurysmal bone cyst of scapula were selected. Based on the CT data of the patient, the scapula (including defect) and pelvis were reconstructed by computer Mimics Medical software. Print out the reconstructed scapula model with a 3D printer. Before operation, the model was used to design the surgical approach and simulate the operation process, to determine the length and Radian of the plate and the number and direction of screws, and to determine the bone mass of the ilium and make reasonable segmentation and distribution. The operation time, the amount of bleeding, the length and Radian of the plate, and the direction and number of screws were recorded.

Results : The average follow-up time was 25.6 months, and none of the 7 patients had recurrence during the follow-up period; The surgical approach, the length and Radian of the internal fixation, the number and direction of screws were consistent with the designed operation plan. The anatomical structure of scapula and the function of shoulder joint gradually recovered.

Conclusions: Compared with traditional methods, the use of 3D printing technology in the treatment of irregular bone tumor bone defect has less trauma, shorter operation time and less bleeding, which can reduce the waste of bone graft and reconstruct the anatomical structure of bone defect more completely.

\section{Introduetion}

Irregular bone includes vertebra, pelvis, jaw and other bones, the scapula is a flat, wide and irregular bone, which also belongs to irregular bone. The bone defects caused by tumors vary in size, shape and irregularity and irregular bones are often located in areas with rich muscle tissue, rich blood supply and deep structure. The traditional method of repair and reconstruction takes a long time and has a large amount of bleeding. During the operation, the steel plate needs to be bent according to the shape and shape of the defect in different parts, so as to further prolong the operation time and increase the amount of blood loss, resulting in the difficulty of anatomical repair and reconstruction of the defect[1]. The 3D printing technology can break through the technical limitations, and can realize the full-scale Structured integration and multi-angle real simulation of the lesion area and the bone structure of the donor site, especially in the repair and reconstruction of bone defects, which can be customized and precise to formulate the repair and reconstruction plan, restore its anatomical structure, muscle attachment point and function to the greatest extent[2].

\section{Patients And Methods}

\subsection{Patient Datas}


Eight patients with scapular ABC treated in our hospital from 2015.01-2019.09 were selected, 1 case of loss of follow-up was excluded, and 7 cases were included in the analysis, including 3 males and 4 females. The age ranged from 18 to 32 years old, the average age was 23.1 years old, and the median age was 22 years old. Compared with the normal contralateral side, 7 patients with the affected side of the shoulder joint had limited shoulder joint movement. The imaging examination of 4 cases showed that the mass penetrated the scapula and the anterior and posterior cortex of the scapular spine, and the oval shape of the shoulder and back of 2 patients the mass is hard, with poor mobility, accompanied by tenderness, the epidermis temperature of the mass is normal, and there is no obvious abnormality in the appearance of the skin. All ABCs in this report were primary lesions and the possibility of metastasis from other parts or system malignant tumors to the scapula was excluded. Figure 1.

This study was reviewed and approved by the ethics committee of our hospital, and all patients agreed and signed the informed consent form.

\subsection{Model Establishment and Treatment}

\subsubsection{Model Building}

Using the patient's preoperative CT data, the patient's scapula (including defect parts) and pelvis models were reconstructed through the computer Mimics Medical modeling software, and the shape, size and volume of the bone defect were accurately measured. According to the data collected and compared with the parameters such as the shape, size and volume of ilium shown by pelvic CT three-dimensional examination, the corresponding donor site of iliac bone defect was accurately selected.

\subsubsection{Print model and simulated surgery}

After the defect model is reconstructed by computer Mimics Medical modeling software, the computer simulated defect repair model and solid defect model are printed and shaped by 3D printer at 1:1. According to the 3D model, pathological results and imaging data, the operation plan was designed. Accurately evaluate the defect location, volume and shape, and determine the length, Radian, screw number and direction of the surgical approach and internal fixation plate; Print out the plate model, then simulate the operation on the 3D printed solid model, and customize the individual anatomical plate. Focus on the internal fixation position and direction of steel plates and screws and determine the best fixation position and direction. Keep practicing until the day of the operation. Figure 2, Figure 3.

\subsubsection{Surgery}

The results of pathological examination in 7 patients showed that all the scapular masses were aneurysmal bone cysts. According to the preoperative plan, an iliac bone equivalent to the size, shape, and/or volume of the defect model is taken and chiseled for use. At the same time, curettage of scapular mass was performed, the bone defects of scapular bone and scapular body were seen during the operation, and the acromion of some patients were floating, the defect was filled with fibrous tissue or blood clot, and pulsatile bleeding or vascular tissue could be seen. The diseased tissue was removed and 
the diseased tissue in the defective area was fully scraped to the normal cancellous bone surface. Trim and polish the iliac bone to basically match the shape of the two defect surfaces, traction the upper limb, place the fracture pieces of the scapula and scapular body, and tamp them. After a good match between the bone and the scapula, the bone is polished with a file to prevent friction against the muscles and fixed with plates and screws. 4 cases of severe bone destruction were treated with curettage and bone grafting (mixed autograft and allograft) of scapular aneurysmal bone cyst, reconstruction and internal fixation of scapula plus iliac osteotomy, 3 patients with small defect volume only underwent curettage of scapular aneurysmal bone cyst, bone graft, reconstruction and internal fixation of scapula and iliac osteotomy. Figure 4.

\subsubsection{Rehabilitation Training and Evaluation of Curative Effect}

Rehabilitation training began gradually 3 days after operation. After discharge, follow-up, shoulder function evaluation and rehabilitation exercise guidance were carried out by means of outpatient revisit.

According to the imaging evaluation of patients 1 year after operation (tumor cavity boundary, bone graft fusion and absorption) and Constant-Murley shoulder joint function score (including pain, daily activities, active range of motion, and muscle strength), the treatment effect is divided into Three levels of excellent, good and poor[3]. Excellent: resume work, tumor cavity boundary disappeared, graft bone resorption was good, $\geq 80$ points $\llbracket$ Good: return to general life and work, tumor cavity boundary is blurred, $60-79$ points; poor: not yet unable to work, tumor cavity boundary is clear, graft bone absorption is poor, $<60$ points. Figure 5,Table 1.

\section{Results}

After discharge from the hospital, follow-up was conducted in an outpatient clinic. The follow-up time was 12-42 months, and the average follow-up time was 25.6 months. No local recurrence or distant metastasis was found in all patients until the last follow-up. The lesion of the scapula has a good appearance, and the plate and screws are well fixed. There are no complications such as infection, internal fixation slippage, broken nails and broken plates, the implanted iliac bone and / or allogeneic bone were gradually absorbed and the function of shoulder joint was gradually recovered. Operation time of 7 patients was $(120 \pm 18)$ min, intraoperative blood loss was $(270 \pm 40) \mathrm{ml}$, the incision approach is consistent with the preoperative plan, and the internal fixation materials used are designed in advance according to the 3D printing model before operation. The amount of bone taken from the iliac bone, the number, position, and curvature of the implants such as the number, position, and curvature of the plate and screws were consistent with the preoperative planning. Among the 7 cases in this group, 6 cases were excellent and 1 case was good.

\section{Discussion}


3D printing technology, also known as rapid prototyping (RP), is a technology for manufacturing physical models by stacking and printing layer by layer; Simply put, it is based on the patient's CT or MRI and other imaging data, the data is processed through computer software to construct a three-dimensional model, and finally a 3D printer is used to print it $[4,5]$. In recent years, with the development of computer science and the innovation of printing technology, the research of 3D printing technology in orthopedics, especially in bone defect repair and reconstruction has become the research hotspot and focus of clinical orthopedics doctors[6]. On this basis, bone tumor specialists combine their 3D printing, trauma orthopedic fracture treatment and tumor bone defect repair and reconstruction treatment concepts, and perform comprehensive and multi-angle evaluations based on three-dimensional images and printed physical models; Develop individualized and precise treatment plans, design surgical approaches, determine the length, arc, number and direction of screws, determine the amount of iliac bone removal, and perform reasonable segmentation and allocation; Improve the safety and accuracy of operation, reduce operation time, trauma and bleeding, and reduce the risk of operation[4]. 3D printing technology is a good combination of digital imaging data and biomaterials, and it is applied to the field of clinical orthopaedic treatment to provide a personalized and accurate surgical scheme for difficult and complex orthopedic diseases, especially for the repair and reconstruction of bone defects[7].

Irregular bone has a special and complex overall morphological structure, different shapes and great differences, and has a multifaceted structure; it is the starting and ending point of many important muscle tissues, surrounded by important nerves and blood vessels and other tissue structures. and most of the irregular bones are located in important functional areas, responsible for a large number of protection of important organs, movement and weight-bearing functions. Therefore, when irregular bone is injured or defective, its special anatomical structure increases the difficulty of treatment. Failure to restore its morphological structure will lead to the loss of some important functions and cause great damage to the spiritual life of patients[8]. Aneurysmal bone cyst is a benign bone tumor-like lesion with unknown etiology, which is highly invasive and destructive[9]. Its first discovery was reported by Jaffe and Lichtenstei et al [10] in 1942. The incidence of ABC accounts for about $1 \%$ of bone tumors, which are mainly concentrated in the long bones of extremities and spine, while irregular bones such as scapula are rare $[9,11]$. Although $A B C$ is a benign bone tumor, its biological behavior is often invasive and easy to relapse. Its invasive and recurrent characteristics often lead to more serious bone destruction, and severe cases can be associated with pathological fractures, which brings some difficulties to treatment[12]. The treatment of aneurysmal bone cysts that occur in irregular bones is more challenging, and the repair and reconstruction of bone defects are also more difficult[11].

The progress of 3D printing technology combined with the innovation of digital imaging equipment has greatly promoted the treatment of irregular bone and other parts of the tumor bone defect. 3D printing technology has outstanding advantages in repairing irregular bone tumor bone defects $\mathbb{\nabla}(1)$ Develop a surgical plan: By using the computer, the location of the peripheral nerves and blood vessels of the focus can be observed in many directions and angles, so as to avoid accidental injury to the nerves and vessels during the operation and accurately measure the volume of the tumor and bone defect, which provides a

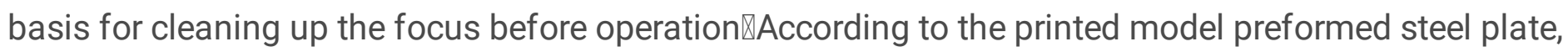


determine the length and Radian of the steel plate and the number and direction of screws, and customize the personalized and accurate operation plan[13, 14]. (2) Simulated operation: Simulate the operation according to the operation plan, constantly improve the operation plan, and determine the best incision position and internal fixation position and direction. So as to reduce the time of operation and the amount of bleeding, reduce the risk of operation and patients' medical expenses. (3) Reduce the risk of surgical operation: The preoperative chief surgeon can use the model to communicate and explain the whole operation process and its important nodes to the team, including anesthesiologists, instrument nurses and internal fixation suppliers, and to simulate the operation. to help the whole surgical team improve their surgical skills and efficiency before operation. During the operation, the model can be put on the operating table after disinfection, and the model can be referred to at any time during the operation, so as to reduce the peeling and injury of soft tissue, reduce the time of operation, reduce the time of intraoperative bleeding, reduce the time of anesthesia, the frequency of use of C-arm and the dosage of antibiotics during operation. to reduce the risk of surgery[14-16]. Stefan Tserovski et al[4] the use of 3D printing technology combined with printed models to assist in diagnosis and treatment in hip revision not only improves the accuracy of diagnosis, but also helps the team to determine the type and size of the prosthesis before operation. (4) Match the donor site ilium: Simulate and match the defect site with the iliac bone through the computer, and accurately select the scapular bone defect site with the same shape or volume as the donor site iliac bone extraction site, and mark the body surface before the operation for faster and more accurate extraction, reducing the operation time and reducing the operation trauma $[17,18]$. (5) Improve the efficiency of doctor-patient communication: In the past, preoperative communication between doctors and patients was mostly based on the patients' own X-ray, CT and other imaging data. For patients and their families without medical knowledge, it is very difficult to fully understand the condition and surgery[5]. Through the computer three-dimensional simulation map and 3D printed solid model, the structure of the real lesion can be presented in front of the eyes, which can more intuitively show the characteristics of the lesion to the patients and their families, explain and explain the treatment plan. so that patients and their families have a clearer understanding of the disease, improve the efficiency of doctor-patient communication, improve the relationship between doctors and patients, but also help patients to cooperate with rehabilitation treatment in the later stage[14, 19].

Therefore, 3D printing technology is applied to the repair and reconstruction of irregular bone tumor bone defects, and the specific three-dimensional physical model can be printed based on the imaging data before the operation to further understand the specific situation of the tumor and bone defect and the distribution of blood vessels around the disease. Choose the appropriate personalized internal fixation, choose the appropriate approach and reduction method, and formulate the best surgical plan; effectively reduce the injury of the muscles, blood vessels and nerves during the operation, reduce the difficulty of the operation, shorten the operation time, and reduce the bleeding during the operation. Improve the safety of the operation, and reduce the damage of the donor site and the waste of the transplanted bone, thereby reducing the incidence of postoperative complications $[1,14,19]$. 
We only use 3D printed prosthesis for simulated surgery and select suitable iliac bones for repair and reconstruction, instead of directly using 3D printed prosthesis for treatment, mainly for the following reasons: (1) The average age of the patients is relatively young and the oldest age is only 32 years old. Compared with taking the discomfort of the iliac bone, directly using 3D printing prosthesis for treatment, the patient's long-term fixation of the foreign body in the body rejects the psychological and mental burden more severe; (2) Although the 3D printed prosthesis is closer to the defect than the iliac bone in the early stage, and it is easier to fix the muscle and other tissue structures, the iliac bone can be more in line with the physiological structure of the human body after long-term plastic transformation, and the muscles are more firmly attached $\mathbb{8}(3)$ Rejection and periprosthetic fractures are inevitable difficulties of the prosthesis. if they are serious, they may need to be treated by a second operation, which aggravates the trauma and economic burden of the patients[20, 21]; (4) The use of 3D printed prosthesis for repair and reconstruction, long-term shoulder joint activities may loosen or even fall off the screws, resulting in insecure fixation of the joints, loosening of the prosthesis, and muscle wear; and complications such as chronic pain are not conducive to postoperative recovery training[22]. Baichuan Wang et al[23] used computer-assisted $3 \mathrm{~d}$ printed hemipelvis prosthesis to treat 11 cases of malignant bone tumors around the acetabulum, and 2 cases showed dislocation of hip prosthesis after surgery. Liang $\mathrm{H}$ et al[24] used three-dimensional printed pelvic prosthesis to reconstruct after resection of pelvic tumor. Of the 35 patients, 7 had delayed wound healing and 2 had hip dislocation. On the contrary, there is no rejection after repair and reconstruction with autogenous iliac bone; after absorption and shaping, the grafted bone can be completely integrated with the scapular bone to achieve bony healing, and the iliac bone and the scapular bone have the same contact area, and the muscle attaches more firmly to the bone surface. At the same time, the complications or adverse reactions such as chronic pain, rejection and prosthesis loosening can be avoided to the maximum extent, and the quality of life of patients can be improved[25].

We hope that the reported cases and treatments can provide a reference option for the repair and reconstruction of tumor bone defects in irregular bone sites. At the same time, this study also has some limitations and shortcomings. First of all, the follow-up time of the patients is short, and there is no comparative study with other treatment methods. Secondly, due to the small sample size, no statistical analysis was carried out. Finally, there are still differences in Constant-Murley scores due to individual factors such as patients' sensitivity to pain and the degree of completion of rehabilitation training. We hope to increase the number of samples, control the range of bone graft, prolong the follow-up time, explore a more objective evaluation system, and carry out statistical analysis in the later work. In the future, we hope to explore and make more use of the advantages of 3D printing technology, and combined with clinical practice to use it in the treatment of more bone tumor-related diseases for the benefit of patients.

\section{Declarations}

Conflict of Interest statement: The authors declare that they have no conflict of interest.

Funding: Nothing to declare 
Ethical approval: "All procedures performed in studies involving human participants were in accordance with the ethical standards of the institutional and/or national research committee and with the 1964 Helsinki declaration and its later amendments or comparable ethical standards."

Informed consent: "Informed consent was obtained from all individual participants included in the study."

Acknowledgment: None.

ORCID number[0000-0002-4725-111X

Total number of words:3163 words(Including abstracts but excluding references and figure legends )

Author contributions: All authors contributed to the study conception and design. Material preparation, data collection and analysis were performed by Guochen Luo, Shuaishuai Chen, Dongyi Li and Mingyang $\mathrm{Yu}$. The establishment of computer model is mainly completed by Xiahua Wang. The first draft of the manuscript was written by Guochen Luo and all authors commented on previous versions of the manuscript. All authors read and approved the final manuscript.

\section{References}

1. Li YT, Hung CC, Chou YC, Chen JE, Wu CC, Shen HC, Yeh TT. Surgical Treatment for Posterior Dislocation of Hip Combined with Acetabular Fractures Using Preoperative Virtual Simulation and Three-Dimensional Printing Model-Assisted Precontoured Plate Fixation Techniques. BioMed research international. 2019;2019:3971571.

2. Moldovan F, Gligor A, Bataga T. Structured Integration and Alignment Algorithm: A Tool for Personalized Surgical Treatment of Tibial Plateau Fractures. Journal of Personalized Medicine 2021, 11(3).

3. Constant CR, Murley AH. A clinical method of functional assessment of the shoulder. Clin Orthop Relat Res 1987(214):160-164.

4. Tserovski S, Georgieva S, Simeonov R, Bigdeli A, Rottinger H, Kinov P. Advantages and disadvantages of 3D printing for pre-operative planning of revision hip surgery. J Surg Case Rep. 2019;2019(7):rjz214.

5. Chuen WK. 3D-printed patient-specific applications in orthopedics. Orthopedic Research Reviews. 2016;Volume 8:57-66.

6. Park JW, Kang HG, Lim KM, Park DW, Kim JH, Kim HS. Bone tumor resection guide using threedimensional printing for limb salvage surgery. Journal of surgical oncology. 2018;118(6):898-905.

7. Ganguli A, Pagan-Diaz GJ, Grant L, Cvetkovic C, Bramlet M, Vozenilek J, Kesavadas T, Bashir R. 3D printing for preoperative planning and surgical training: a review. Biomed Microdevices. 2018;20(3):65.

8. Alexiou KI, Roushias A, Varitimidis SE, Malizos KN. Quality of life and psychological consequences in elderly patients after a hip fracture: a review. Clin Interv Aging. 2018;13:143-50. 
9. Mascard E, Gomez-Brouchet A, Lambot K. Bone cysts: unicameral and aneurysmal bone cyst. Orthop Traumatol Surg Res. 2015;101(1 Suppl):119-27.

10. Lichtenstein L, Jeffe HL. Eosinophilic granuloma of bone: With report of a case. Am J Pathol. 1940;16(5):595.

11. Jamshidi K, Haji Agha Bozorgi M, Hajializade M, Bagherifard A, Mirzaei A. Tailored treatment of aneurysmal bone cyst of the scapula: en bloc resection for the body and extended curettage for the neck and acromion. J Shoulder Elbow Surg. 2020;29(5):961-7.

12. Chebib I, Chang CY, Lozano-Calderon S, Nielsen GP. Histopathology of fibro-osseous and cystic tumors of bone. Diagnostic Histopathology 2020.

13. Dugarte AJ, Tkany L, Schroder LK, Petersik A, Cole PA. Comparison of 2 versus 3 dimensional fracture mapping strategies for 3 dimensional computerized tomography reconstructions of scapula neck and body fractures. Journal of orthopaedic research: official publication of the Orthopaedic Research Society. 2018;36(1):265-71.

14. Senkoylu A, Daldal I, Cetinkaya M. 3D printing and spine surgery. J Orthop Surg. 2020;28(2):2309499020927081.

15. Guo F, Dai J, Zhang J, Ma Y, Zhu G, Shen J, Niu G. Individualized 3D printing navigation template for pedicle screw fixation in upper cervical spine. PLoS One. 2017;12(2):e0171509.

16. Marconi S, Pugliese L, Botti M, Peri A, Cavazzi E, Latteri S, Auricchio F, Pietrabissa A. Value of 3D printing for the comprehension of surgical anatomy. Surgical endoscopy. 2017;31(10):4102-10.

17. Jang WH, Lee JM, Jang S, Kim HD, Ahn KM, Lee JH. Mirror Image Based Three-Dimensional Virtual Surgical Planning and Three-Dimensional Printing Guide System for the Reconstruction of Wide Maxilla Defect Using the Deep Circumflex Iliac Artery Free Flap. J Craniofac Surg. 2019;30(6):182932.

18. Vehmeijer M, van Eijnatten M, Liberton N, Wolff J. A Novel Method of Orbital Floor Reconstruction Using Virtual Planning, 3-Dimensional Printing, and Autologous Bone. J Oral Maxillofac Surg. 2016;74(8):1608-12.

19. Wu WY, Xu WG, Wan CY, Fang M. Preoperative Plan with 3D Printing in Internal and External Fixation for Complex Tibial Plateau Fractures. Orthopaedic surgery. 2019;11(4):560-8.

20. Abdel MP, Watts CD, Houdek MT, Lewallen DG, Berry DJ: Epidemiology of periprosthetic fracture of the femur in 32644 primary total hip arthroplasties: a 40-year experience. The bone \& joint journal 2016, 98-b(4):461-467.

21. Giaretta S, Momoli A, Porcelli G, Micheloni GM. Diagnosis and management of periprosthetic femoral fractures after hip arthroplasty. Injury. 2019;50(Suppl 2):29-s33.

22. Wang B, Wu Q, Liu J, Chen S, Zhang Z, Shao Z. What are the Functional Results, Complications, and Outcomes of Using a Custom Unipolar Wrist Hemiarthroplasty for Treatment of Grade III Giant Cell Tumors of the Distal Radius? Clin Orthop Relat Res. 2016;474(12):2583-90.

23. Wang B, Hao Y, Pu F, Jiang W, Shao Z. Computer-aided designed, three dimensional-printed hemipelvic prosthesis for peri-acetabular malignant bone tumour. Int Orthop. 2018;42(3):687-94. 
24. Liang H, Ji T, Zhang Y, Wang Y, Guo W. Reconstruction with 3D-printed pelvic endoprostheses after resection of a pelvic tumour. Bone Joint Journal. 2017;99-B(2):267.

25. Xu S, Niu F, Chen Y, Jin Q, Gui L. Repairing Cranial Defect With Autogenous Mandibular Outer Plate. J Craniofac Surg. 2020;31(6):1768-72.

\section{Tables}

Due to technical limitations, table 1 and 2 is only available as a download in the Supplemental Files section.

\section{Figures}
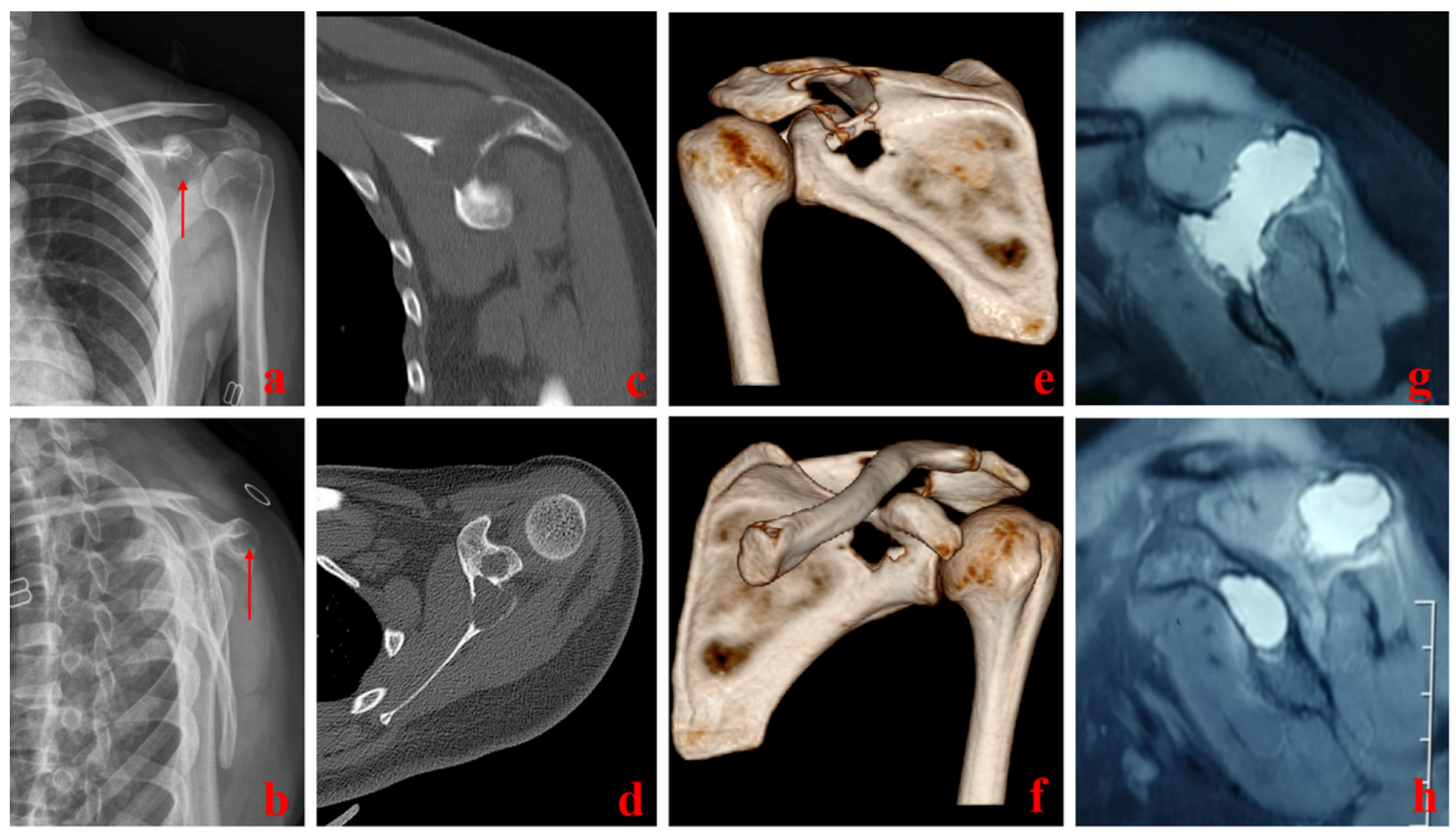

\section{Figure 1}

Figures $\mathrm{A}$ and $\mathrm{B}$ are $\mathrm{DR}$ anteroposterior and lateral radiographs. Low density shadow can be seen in the scapular area with a clear boundary and a sclerosing zone on part of the edge. Figure $C$ and $D$ show irregular and cystic bone destruction of CT, scapular spine and scapular neck in cross-sectional and sagittal position, respectively, and the bone cortex is punctured before and after perforation. Figure $\mathrm{E}$ and F show three-dimensional reconstruction of the CT scapula, showing severe destruction of the scapula, especially the scapular bone, and penetrating the anterior and posterior bone cortex. Figure $\mathrm{G}$ and $\mathrm{H}$ are T2 images of MRI, showing patchy abnormally high signal and lobulated images. 

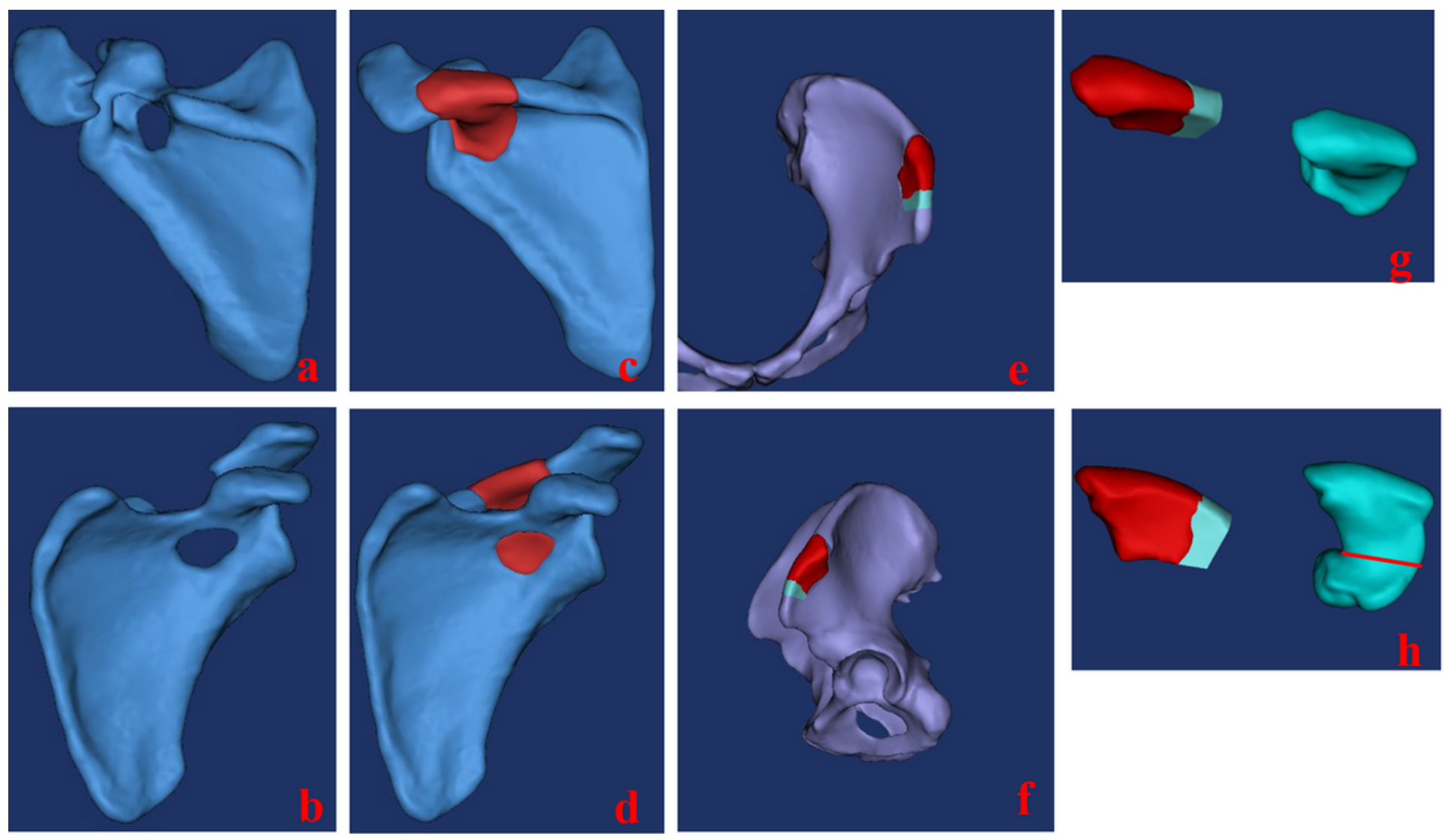

Figure 2

Figures $A$ and $B$ show the reconstruction model of the affected side of the scapula, and the site of bone destruction can be seen obviously. Figures $C$ and $D$ show the reconstruction of the defect area of the scapula. Figures $\mathrm{E}$ and $\mathrm{F}$ show a simulation of the iliac bone, in which the red part will be used to repair the scapular spine and the green part will be used to fill the gap. Figure $\mathrm{G}$ and $\mathrm{H}$ show the defect area and the proposed ilium, the red and green on the left is the ilium to be taken, and the green defect area on the right is reconstructed; the red line in the green part on the right in figure $\mathrm{H}$ is the boundary, and the above part is consistent with the shape of the red part of the ilium defect, so as to amputate the ilium for scapula repair. 

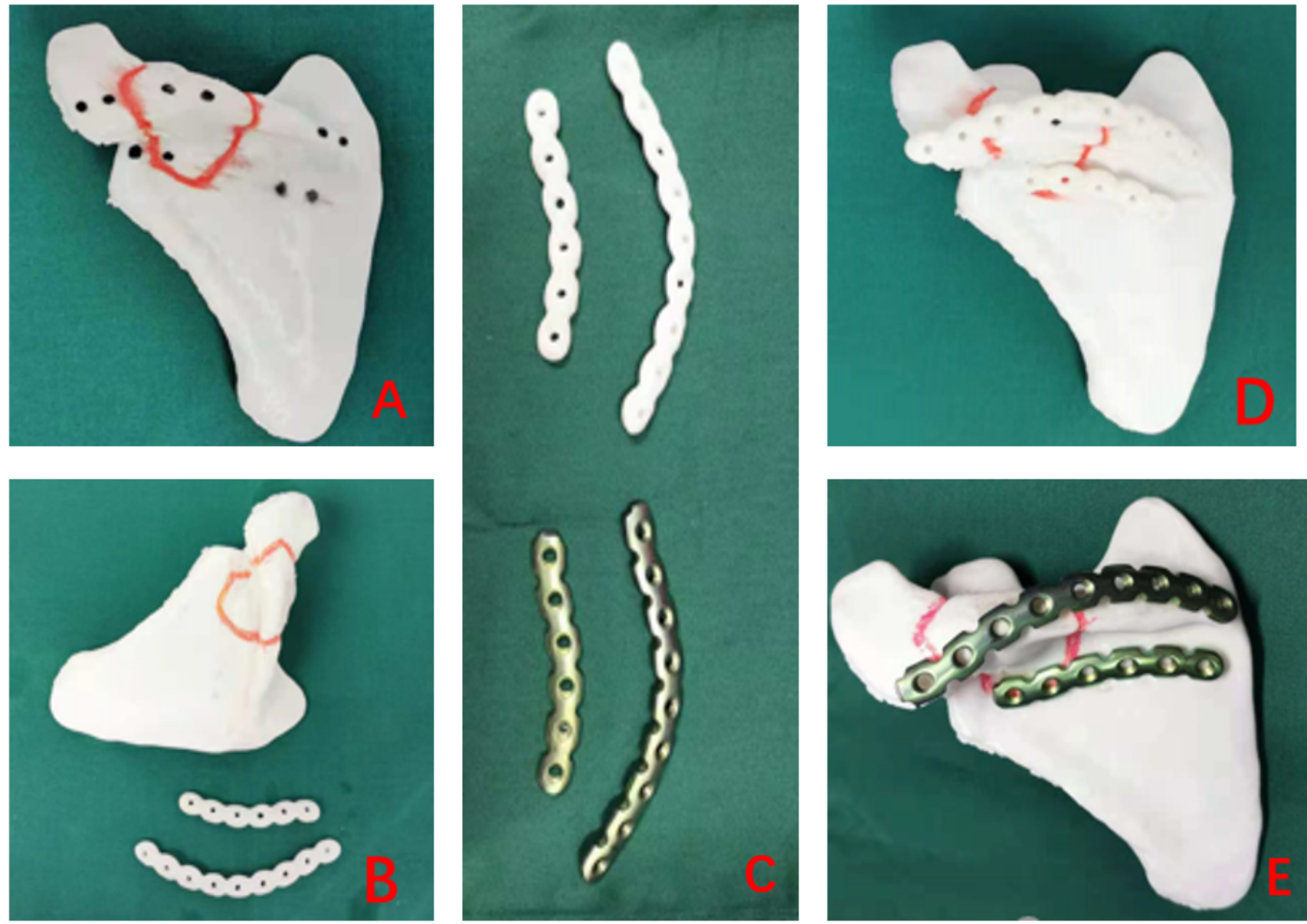

\section{Figure 3}

Figures $\mathrm{A}$ and $\mathrm{B}$ show the print model, in which the red area is the reconstructed bone destruction area and the black mark is the simulated screw fixation position. Figure $\mathrm{C}$ shows the prefabricated steel plate according to the model. Figures $\mathrm{D}$ and $\mathrm{E}$ are simulated and fixed on the model. 

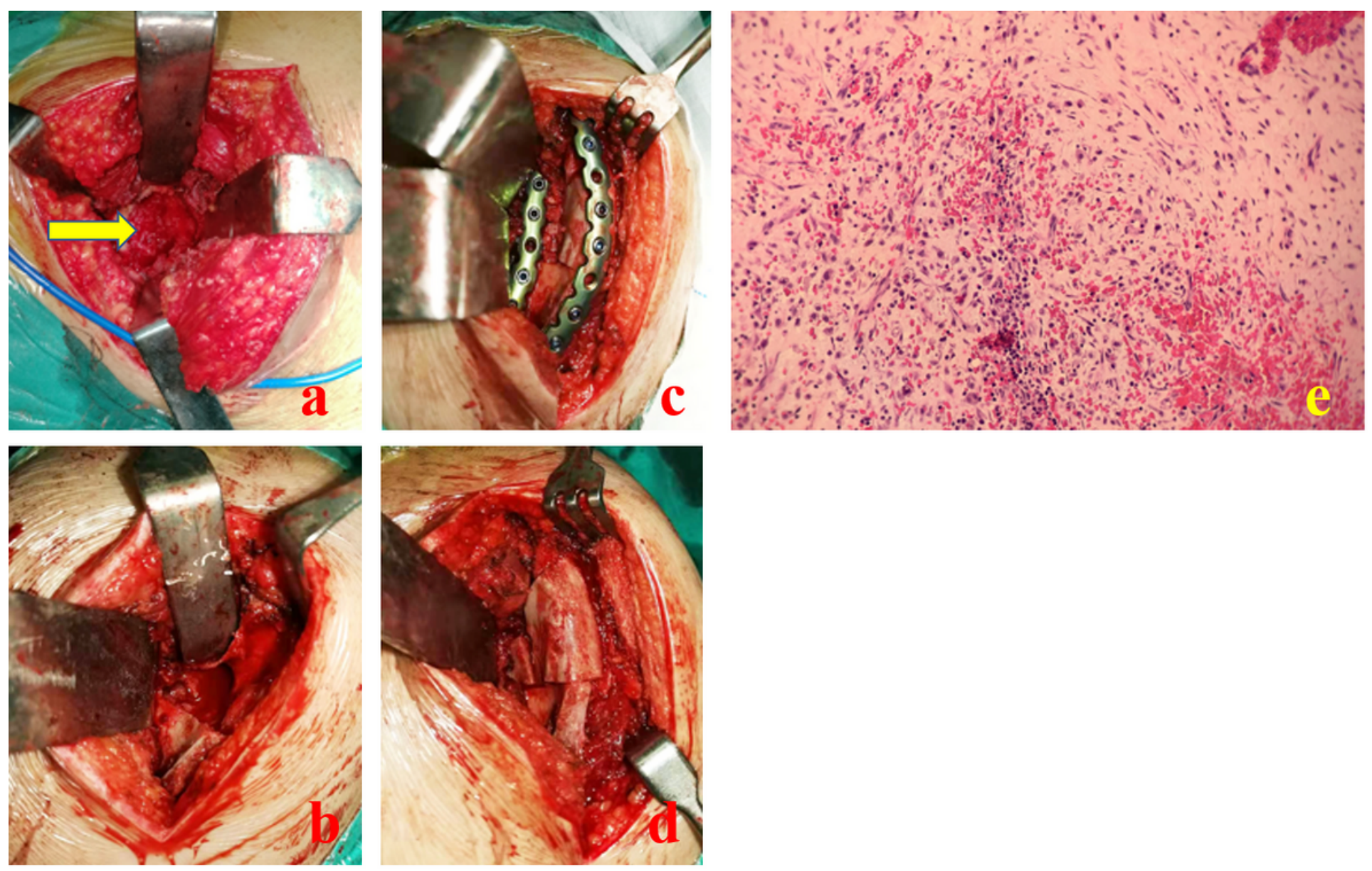

\section{Figure 4}

The yellow arrow in figure $A$ refers to the focus, figure $B$ shows the lesion after curettage, figure $C$ shows the iliac bone matching the scapular defect area, and figure $D$ shows the plate and screw fixation. Figure E is a pathological examination, showing fibrous connective tissue, yellow iron-containing blood and multinucleated giant cells. 

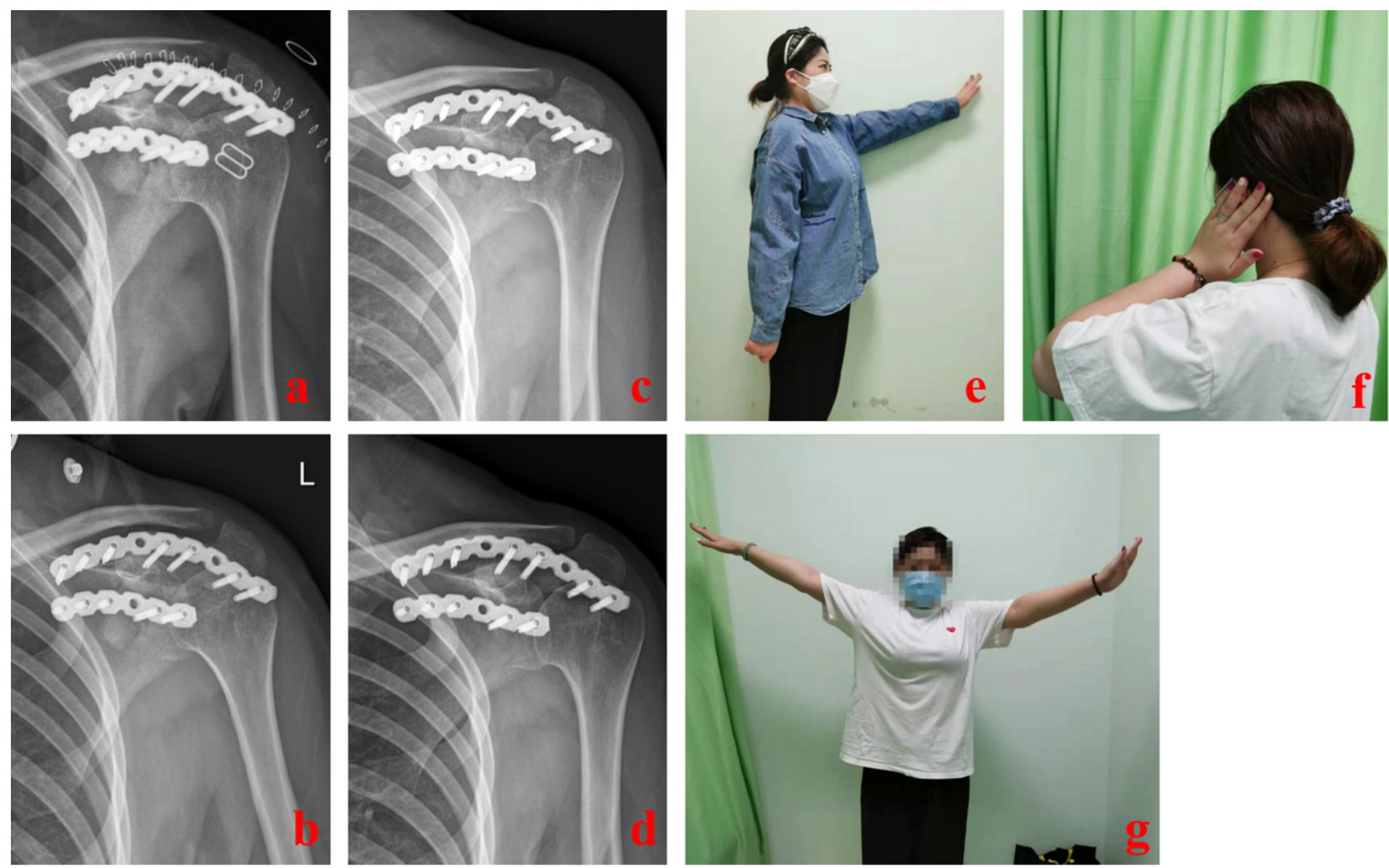

\section{Figure 5}

Figure A, B, C and D are DR films reexamined at 1 week, 1 month, 3 months and 12 months after operation, respectively. It can be seen that the density of the bone graft area of defect repair and reconstruction decreases gradually, and at 12 months, the density is the same as that of the surrounding normal cancellous bone. There is no obvious boundary between the tumor cavity and the surrounding normal bone tissue, and the tumor cavity basically disappears. Pictures $E, F$ and $G$ show the functional recovery of the patients one year after operation, showing the gradual recovery of shoulder abduction, external rotation and protrusion function of the patients.

\section{Supplementary Files}

This is a list of supplementary files associated with this preprint. Click to download.

- Table1.docx

- Table2.docx 